\title{
Design and Implementation of Scenic Area Information Service System based on Semantic loT \\ Hai-Chao YANG ${ }^{1, \text { a }}$,Rui Guo ${ }^{1}, \mathrm{Jun}_{\mathrm{Li}}{ }^{2}$
}

\author{
${ }^{1}$ College of Mathmatic and Computer,Dali University,Dali 671003,China \\ ${ }^{2}$ Yunnan Luwutong Technology Co.Ltd,Dali,Yunnan 671000,China \\ aemail: dlyhc@163.com
}

Keywords: wisdom scenic; Internet of things; semantic network; wisdom tourism

\begin{abstract}
Aiming at characteristics of wisdom scenic, based on semantics Internet of things technology, we have realized a kind of scenic intelligent information service system which integrates various functions including scenic guide, environmental information perception, etc in one, designed system architecture based on semantic Internet of things, put forward ontology model of wisdom scenic which integrates various information including sensors, scenic spots, scenic facilities, and so on. And we have introduced reasoning process and rules based on wisdom scenic ontology, designed a kind of attractions smart hardware which integrates various sensors, realized prototypes of software system and smart hardware. The experiment shows that the system can provide personalised and intelligentized information service for tourists effectively.
\end{abstract}

\section{Introduction}

According to application requirements of wisdom scenic, the paper researches and develops a kind of intellectualized system which has higher integration level, integrates various functions into one including scenic spot information transmission, environmental data collection, etc, integrates tourists' mobile phone APP seamlessly and provides comprehensive and real-time information of each scenic spot inside scenic regions for tourists. Aiming at heterogeneity and diversity characteristics of scenic spot information, we adopt matter association technology of semantic network, establish ontology models of wisdom scenic, integrate various information of scenic spots and provides intelligent information service for tourists through semantic reasoning.

\section{Related Research}

Aiming at diversity and isomerism problems of tourism information, a lot of researchers have launched research based on tourism information system of semantic network. Yang Qingyun[1] and other people have put forward the method of adopting semantic web technology constructs tourism information service platform to improve precision ratio and recall ratio of tourism domain search. Feng Xin[2] and other people have designed a kind of tourism information system based on ontology, defined tourism ontology and tourist ontology and realized semantic query and intellectual service of tourism information. Hung-Yu Chien[3] and other people have designed and realized exhibition tour and recommendation system based on zigbee and ontology, constructed ontology for Sun Moon lake, defined SWRL rule and realized knowledge reasoning under tourism scene.

The W3C Semantic Sensor Network Incubator group (the SSN-XG) produced an OWL2 ontology to describe sensors and observations[6].

Among the above researches, one is to combine semantic web technology and tourism domain. The other is to combine semantic web technology with Internet of things. Few researchers launch researches which combine wisdom scenic field with semantic network and Internet of things at the same time. 


\section{System General Structure}

Core of "wisdom scenic" is to realize all present and real-time perception and visual management towards scenic resources and environment and tourists' activities, etc through integration of information technology including sensing network, Internet of things and Internet. According to the aim, combined with semantic network and facing resource framework (ROA), the whole system is constituted by perception layer, data layer, semantic layer, resource representation layer and application layer. As shown in figure 1.

Semantic layer: based on semantic data in data layer, it finishes expansion query and reasoning based on semantics including semantic query preprocessing, semantic concept extension algorithm, ontology reasoning and query and inquiring language and returning to query access end with SPARQL.

REST resource representation layer: based on software framework of surface resources, it provides stateless REST style interface for application layer to let other application systems including mobile phone APP, scenic spot smart box, etc invoke server resource information resources in a stateless approach conveniently.

Application layer: it is mainly tourists phone APP. It provides mobile phone application software of scenic area intelligence service. It has functions of self-help tour, attractions information acquisition, personalized recommendation of scenic spots, online booking, and so on.

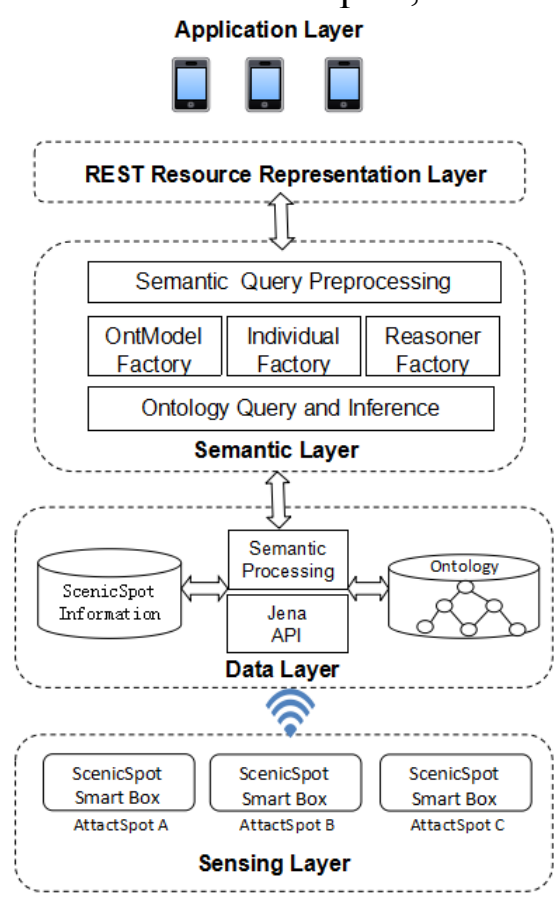

Fig.1 overall structure

\section{Hardware System Design}

\section{Scenic Spot Smart Box}

Aiming at application scenarios of wisdom scenic, the paper has researched and developed a kind of smart hardware devices---scenic spot smart box. Based on perception layer of Internet of things, hardware part all integrates into scenic spot smart box, which is constituted with five parts including single chip microcomputers, sensors, bluetooth module, power module and intelligent gateway. The hardware prototype of scenic spot smart box is shown as figure 2 .

Sensors are directly connected to I/O interfaces of singlechips. After collecting sensor data, singlechips communicate with intelligent gateways and transmit data to gateways through bluetooth modules. Gateways can upload data to severs with WiFI or GPRS. At least one smart box with gateway function is placed in each scenic spot. The middle is center node of the scenic spot. Scenic 
spots can place multiple boxes without gateway function at the same time according to practical situation. These boxes and center nodes constitute star network and transmit data to intelligent gateways of center nodes. Wireless communication adopts bluetooth 4.0, which has advantages of low power consumption, low latency, ultralong connection distance, and so on. Transmission distance can reach more than one hundred meters[4]. More than one box are placed in different locations in the same scenic spot to collect data and enhance data accuracy of the whole scenic spot.

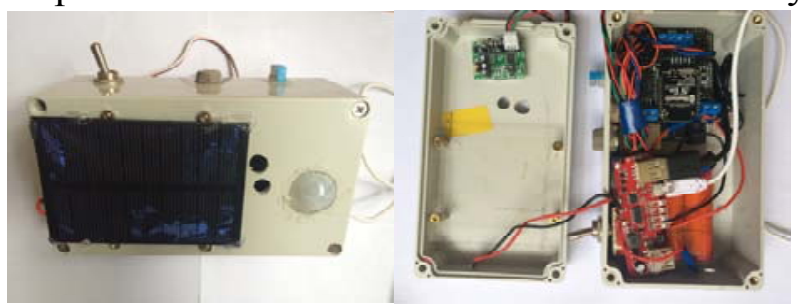

Fig.2. prototype of scenic spot smart box

General structure of hardware system is shown as figure 3. Main modules contain:

(1) Arduino Main Control Panel

Arduino main control panel adopts Arduino Leonardo. It is a micro-controller board based on ATmega32u4. It inputs/ outputs pins with twenty figures. Thereinto, seven pins are used for PWM output and twelve pins are used for analog input[6]. It has characteristics of small size and low price.

(2) Sensor

Scenic spot smart box integrates DHT11. Digital temperature and humidity sensors are used to collect scenic spot temperature and moisture data. MQ2 smoke transducers are used to collect smoke in the air of scenic spots. PD4NS air dust sensors are used to detect PM2.5 data of scenic spots. MIC sound transducers are used to detect sound intensity in surrounding environment. Body heat release motion sensors compute visitors flow rate through detecting human movement.

(3) Bluetooth communication module

Bluetooth communication module adopts HC-06 wireless bluetooth serial interface, passthrough and slave module. It connects through TX/RX interfaces of Arduino. The biggest advantage of the module is low price and can carry out bluetooth communication with Android mobile phones in slave mode conveniently.

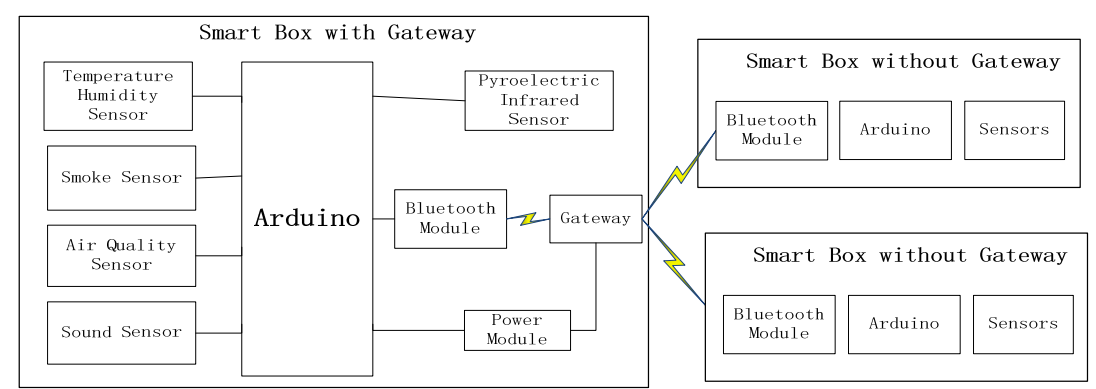

Fig.3. overall structure of scenic spot smart box

\section{Power Module}

In order to solve the problem that alternating current can't reach certain scenic spots, scenic spot box adopts two kinds of power supply modes including alternating current and solar power supply. It is supplied in the way of modules. We can choose to install solar energy module or alternating current module of boxes to adapt to different power supply environment. Figure 4 is composition structure of solar energy power supply module. 


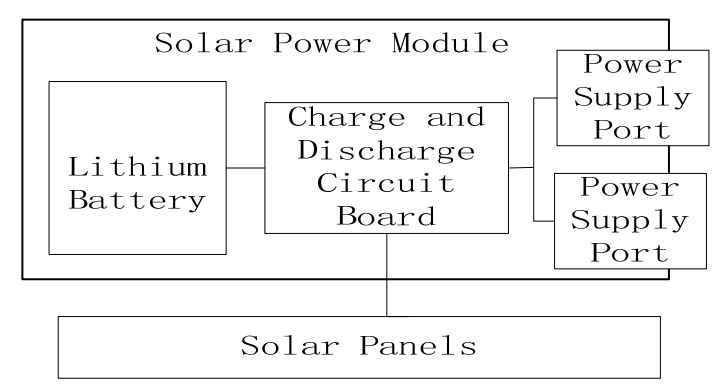

Fig.4. power module

\section{Ontology Model of Wisdom Scenic}

Besides tourism domain information of tourists, scenic spots, culture, etc, wisdom scenic also has a large number of sensor data, such as: temperature, illuminance, human movement, etc. In order to realize mutual operation between two types of information, we integrate tourism domain concept and sensor information, create ontology of wisdom scenic with OWL including concept of scenic area and its relationship. Ontology structure is shown as figure 5.

Scenic spots, shopping, accommodation, catering, traffic, routes, historical culture, weather, public facilities, location and intelligent equipment, etc are main class one of ontology. Subclasses are established under class one. Each subclass has property, restrain and incidence relation. Concrete data of subclasses are examples of ontology. The class of public facilities contain subclasses of toilets, signboards, dustbins, public telephone booths, leisure chairs, parking lots, etc. The class of intelligent device is IT equipment of Internet of things, Internet, etc which are installed inside wisdom scenic, including subclasses of scenic spot wisdom boxes, sensors, video surveillance, etc. Its example is environmental data collected from perception layer. Position class contains five subclasses of roads, bus stations, subway stations, GPS Coordinates and regions[8]. All scenic spots, accommodation, public facilities, sensors, etc inside scenic spots have position information. These subclasses have incidence relation with position subclass.

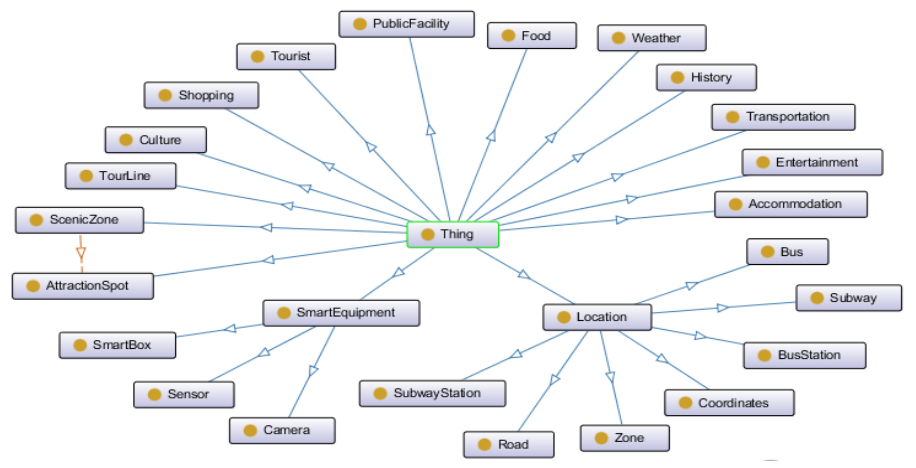

Fig.5. ontology of wisdom scenic

\section{Ontology Reasoning of Wisdom Scenic}

\section{Reasoning Process}

After establishing ontology class, ontology property and ontology instance of wisdom scenic, we can provide more intelligent personalized service in information services of tour guide, guide to visitors, shopping guide, etc of scenic spots for tourists with ontology reasoning. The paper makes use of API reasoning toolkit. Jena inference API supports ontology languages of RDF and OWL. Through ontology class, ontology instance and reasoning rule, we can infer hidden information with inference engine and carry out semantic query with SPARQL. In wisdom scenic spots[9], because it exists real-time context information of tourists, sensors, etc, we need to carry out real-time semantic 
annotation towards these information. The overall structure of reasoning subsystem is shown as figure 6. Sensors Repository is real-time data of sensors stored in relational database. Tourism Repository is tourism information stored in relational database including tourists, scenic spots, accommodation, and so on. Based on ontology model defined in advance, we generate the two kinds of data into ontology instance through Individual Factory, Thus, we realize semantic annotation towards scenic spot information, mix sensor data and tourism domain information together and provide semantic data support for reasoning based on context sensing.

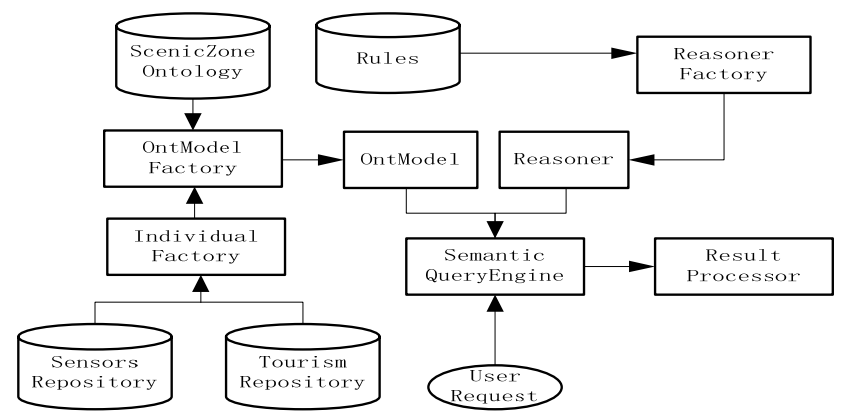

Fig.6. Structure of inference subsystem

Reasoning steps are: (1) read-in ontology defined with OWL language; (2) reading related data from relational database and setting up ontology instance; (3) read-in inference rule base; (4) creating inference machine; (5) importing ontology model, inference machine and user query requests to semantic query engine for semantic reasoning and query; (6) providing query results to Result Processor to handle.

\section{Structure of Wisdom Scenic Inference Rules}

The paper takes internal rule engine of Jean as inference machine. Jena has built in forward chained reasoning RETE engine and Tabled Datalog engine. The premise that inference machine can get reasoning results is that we need to construct reasoning rules. Jena has provided general rules of transmission, mutual inverse, non-overlapping, etc. The paper constructs self-defined inference rules aiming at wisdom scenic, including the following two categories:

\section{Reasoning Rule Based on Tourism Domain Knowledge}

Based on inference rule of tourism domain knowledge construction including scenic spots, accommodation, shopping, traffic, etc, we provide knowledge inference in aspects of tour guide, shopping guide and tour when tourists are visiting in scenic spots. Next, we illustrate this category of inference rule:

(1) [Rule1:(?x isNearBy ?y)(?y hasFacility ?z) $\rightarrow($ ?x isNearBy ?z)]

If $x$ nears $y$ and $y$ has public facility $z$, well, $x$ nears pubic facility $z$. For example, view spot $A$ nears view spot $B$, there is leisure pavilion $C$ (public facility) in view spot $B$, well, the leisure pavilion $\mathrm{C}$ nears view spot $\mathrm{A}$. The rule can help tourists get nearby public facilities information.

(2) [Rule2:(?x hasAttractSpot ?y)(?z isLocatedIn ?y) $\rightarrow($ ?x hasAccomadation ?z)]

If scenic spot $x$ has view spot $y$ and the hotel $z$ is located within view spot $y$, we can deduce that scenic spot $\mathrm{x}$ has the hotel $\mathrm{z}$.

(3) [Rule3:(?x isInterestingIn ?y)(?z hasAttractionType ?y) $\rightarrow($ ?z canFitFor ?x)]

If tourist $\mathrm{x}$ is interested in view spot $\mathrm{y}$ and the type of view spot $\mathrm{z}$ is $\mathrm{y}$, view spot $\mathrm{z}$ is suitable for tourist $\mathrm{x}$.

\section{Inference Rule Based on Context Sensing of Scenic Spots}

Contextual information of scenic spots not only contains real-time information of temperature, noise, visitors flow rate, etc from sensors, but also contains information of location, network condition, etc from tourists' mobile phones. Based on these rules of context information construction, we can infer according to real-time condition of view spots and tourists and thereby promote intelligentization and individuation of scenic area information service. Examples of this 
kind of rules are as follows:

(1) [Rule4:(?x hasAge ?a) greaterThan(?a,60) (?z isInstalledIn ?y)(?z hasCrowdDensity ?k) lessThan $(? \mathrm{k}, 0.3) \rightarrow($ ?y canFitFor ?x)]

If tourist $\mathrm{x}$ 's age is over sixty; smart box $\mathrm{z}$ is installed in view spot $\mathrm{y}$ and human flow density value of smart box $\mathrm{z}$ is lower than 0.3 people $/ \mathrm{m} 2$, we can infer that view spot $y$ is suitable for tourist $\mathrm{x}$. Through the rule, we can recommend view spots with relatively low human flow density for the old.

(2) [Rule5:(?x isInstalledIn ?y)(?x hasLightIntensity ?k) greaterThan(?k,10000) $\rightarrow$ (?y isGuidedBy “audio")]

If sensors are installed in view spot y and light intensity value of sensor x exceeds 10,000 , well, navigation information type provided by view spot $y$ is voice frequency. View spot $y$ is under the sun for the moment and illuminance is too strong. When provide navigation information for tourists, we shouldn't take the way of using displayers including videos, pictures, etc and we should take voice frequency as the principle thing.

\section{Conclusion}

Aiming at the field of wisdom scenic, the paper has designed and realized a kind of intelligent information service system based on semantic Internet of things technology. It is constituted by three parts including smart hardware based on Internet of things-attractions notification box, server software based on semantic website and smartphone APP. Besides functions including environmental information perception, scenic spot self-help tour, etc, based on semantic web technology, the system mix tourism domain knowledge and real-time data of scenic spots collected by sensors together to infer and provide intelligent information service based on contextual information perception of view spots for tourists.

At present, we have only realized prototype system. We still need to strengthen research on operational ease furthermore and promote the system's degree of automation to adapt to practical application of wisdom scenic.

\section{Acknowledgment}

In this paper, the research was sponsored by the Technological Innovation Powerful Province Planned Project of Yunnan Province(Project No.2014AA006),Scientific Research Fund Project of Yunnan Education Department(Project No.2015Z157) and 2014 Application Development Research Fund Project of Dali University.

\section{References}

[1]YANG Qing-yun.Research on the Tourism Information Service Platform Based on Semantic Web.Journal of Shaoyang University,2014(06):19.

[2]FENG Xin.Applications of Ontology for Tourism Information System.2010(3):128-130.

[3]Hung-Yu Chien,Shyr-kuen Chen.Design and Implementation of ZigBee-Ontology-Based Exhibit Guidance and Recommendation System. International Journal of Distributed Sensor Net works. Volume 2013, Article ID 248535,8 pages.

[4]YUAN Ling-yun,WANG Xing-chao.Survey of Semantics of Things.Computer Science,2014 (06):239-243.

[5]Pfisterer et al.SPITFIRE:Towards a Semantic Web of Things.IEEE Commun. Mag., 49 (11) (2011), pp. $40-48$.

[6] Arduino. Arduino Leonardo. 2013,http://arduino.cc/en/Main/ArduinoBoardLeonardo. 